\title{
The Evolution of Silicosis into Massive Pulmonary Fibrosis - Case Series
}

\author{
Enikő Székely-Vass', Zsuzsánna Szász',2, Gyopár Horváth¹, Mădălina Hozoi \\ 1 Department of Occupational Medicine, Mureș County Hospital, Tîrgu Mureș, Romania \\ 2 Department of Occupational Medicine, University of Medicine and Pharmacy, Tîrgu Mureș, Romania
}

\section{CORRESPONDENCE}

Zsuzsánna Ágnes Szász

Bld. 1 Decembrie 1918 nr. 28

Tîrgu Mureș, Romania

Tel: +40745609665

E-mail: zsuzsannaagnes@yahoo.com

\section{ARTICLE HISTORY}

Received: December 21, 2017

Accepted: February 15, 2018
Enikő Székely-Vass • Blv 1 Decembrie 1918 nr. 28 540011 Tîrgu Mureș, Romania. Tel: +40 265263585 Gyopár Horváth • Blv. 1 Decembrie 1918 nr. 28, 540011 Tîrgu Mureș, Romania. Tel: +40 265263585

Mădălina Hozoi • Blv. 1 Decembrie 1918 nr. 28, 540011 Tîrgu Mures, Romania. Tel: +40 265263585

\begin{abstract}
Silicosis is one of the oldest occupational diseases, known since the time of Hippocrates, and the diagnosis can be established after a latency time of more than 10 years after exposure cessation. In some cases of chronic silicosis, the disease can progress slowly, evolving over several decades, but it can also evolve into massive pulmonary fibrosis, also known as accelerated silicosis. Furthermore, in extremely rare cases, the disease can present a rapid progression into acute silicosis or silicoproteinosis. The present article gives a deeper view into silicosis and secondary massive progressive pulmonary fibrosis, as well as the difficulties encountered in the management of such patients. We present two different but similar cases that underline the importance of a precise diagnosis, pointing out the long latent period after the exposure to silicon-dioxide, more than 15 years in both cases. This accelerated form of silicosis associated with massive pulmonary fibrosis progressed in 2 years into respiratory failure in both cases.
\end{abstract}

Keywords: silicosis, massive pulmonary fibrosis, Caplan's syndrome, large pulmonary mass

\section{INTRODUCTION}

Silicosis is one of the oldest occupational diseases, known since the time of Hippocrates, ${ }^{1}$ and it can lead to lung disease due to inhalation of dusts containing silicon-dioxide crystalline $\left(\mathrm{SiO}_{2}\right)$ after a long period of exposure - usually more than a decade. The diagnosis can be established after a latency time of more than 10 years, even after exposure cessation. ${ }^{2}$

The reaction of the pulmonary tissue to inhaled dusts is characterized by permanent alteration or destruction of normal alveolar structure and interstitial lung collagen-type reaction. The developed interstitial lung disease is irreversible and progresses due to the permanent fibroblast activity. ${ }^{1,2}$

From a radiological point of view, there are several stages of this disease: (1) the presilicotic stage with increased hilar opacities associated with trabecular thickening; (2) progressive interstitial fibrosis, extended hilar shadow to the lung periphery; (3) typical silicosis nodules with $1 \mathrm{~mm}$ diameter, which appear disseminated around the hilus. Subsequently, these nodules can grow up to 1-3 mm in diameter and can disseminate throughout the whole lung, or they can increase 
to $10 \mathrm{~mm}$ in diameter; (4) finally, the confluent nodules can appear in different forms (round, ovoid, or irregular bilateral-pseudo appearance). Other radiological modifications may include pleural thickening, emphysema, or bronchiectasis, or the lesions can be described as large masses on the thoracic radiography. Lung abscesses can form due to infections such as tuberculosis or mycosis. The enlarged lymph nodes may later present peripheral calcifications. ${ }^{3-5}$

In some cases of chronic silicosis, the disease can progress slowly, evolving over several decades, but it can also evolve into massive pulmonary fibrosis, also known as accelerated silicosis. Furthermore, in extremely rare cases, the disease can present a rapid progression, into acute silicosis or silicoproteinosis.

The main occupations in which subjects are exposed to silica dusts include mining (ferrous, coal, silicates, hydro tunnel workers, railway workers); industrial workers (sandblasters, welders); workers in the production of acidresistant bricks; workers in porcelain/tiles industry or any kind of jobs with $\mathrm{SiO}_{2}$ dust exposure.2

Bronchoalveolar lavage is a less invasive method that allows the extraction of cells and proteins that are present on the epithelial surface of the lower respiratory tract, to properly establish a diagnosis of pulmonary disease. ${ }^{6}$

The diagnosis of occupational respiratory diseases is based on the patient's clinical history of exposure and on the chest X-ray. 7,8

In rare cases, pulmonary silicosis can occur in patients with preexistent interstitial lung disease such as rheuma- toid arthritis with pulmonary fibrosis. Occupational exposure in such cases will lead to the so-called "Caplan's syndrome”, which is defined as rheumatoid pneumoconiosis. ${ }^{1,2,9}$

Several studies have confirmed that the serum activity of angiotensin-converting enzyme (ACE) is increased in patients with silicosis, coal worker's pneumoconiosis, and sarcoidosis, and its elevated serum levels can indicate disease progression and severity. ${ }^{10}$

\section{CASE SERIES}

The manuscript presents a series of two clinical cases of patients with occupational pulmonary silicosis with consequent massive progressive pulmonary fibrosis, presenting with clinical symptoms long after cessation of exposure. All patients consented to publication of their data and the publication was approved by the Ethics Committee. All the examinations were performed in accordance to the principles stated in the Declaration of Helsinki.

The first case is a 43-year-old male patient, smoker, who has been a professional underground miner for 18 years, out of which 12 years in a copper mine. From the medical history it is noticeable that the patient was diagnosed with seropositive rheumatoid arthritis in 2013. Initially, the patient had been administered methotrexate for 6 months, which required cessation due to extensive hepatic cytolysis, and the replacement therapy included Sulfasalazine administration. During the patient's first hospitaliza-
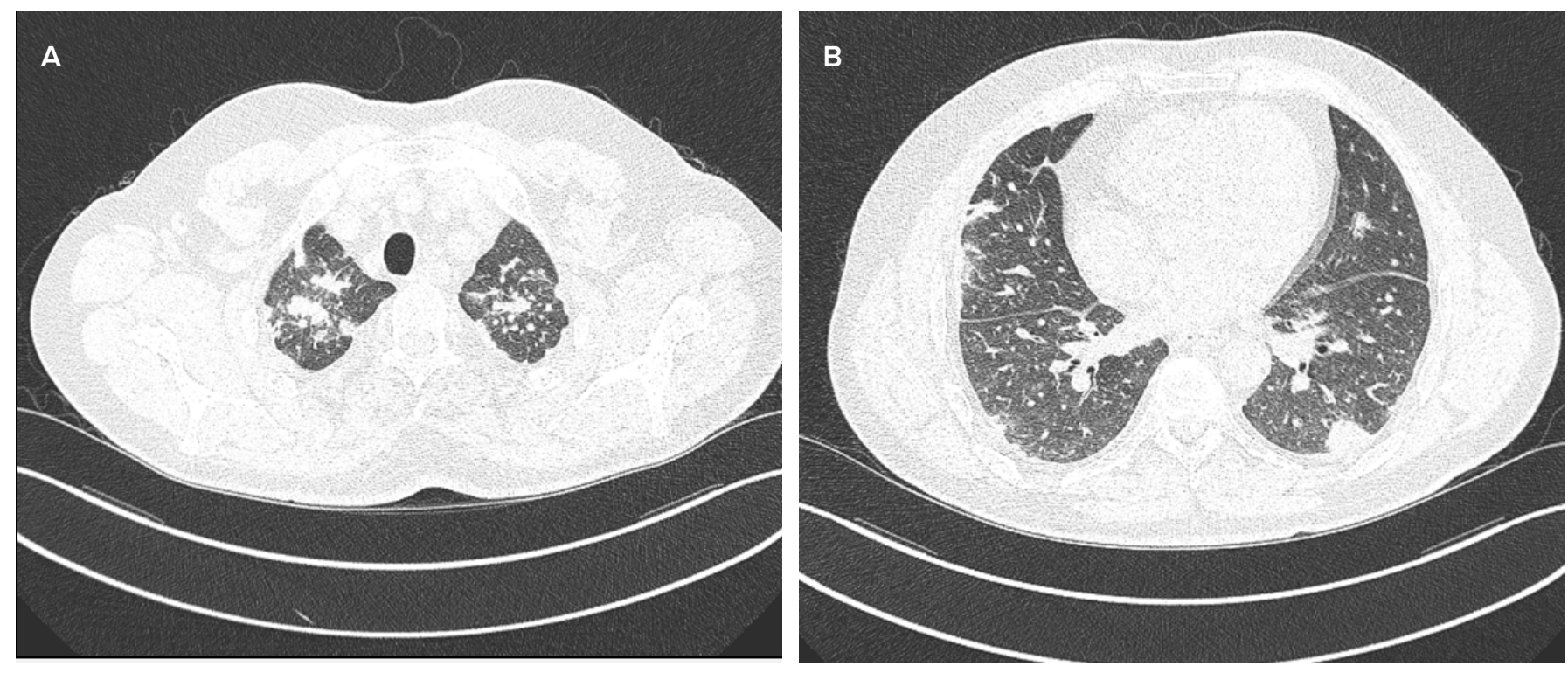

FIGURE 1. A, B - Thoracic CT images showing multiple pulmonary nodules between $1-5 \mathrm{~mm}$ in diameter, some located at the peripheral region with $15 \mathrm{~mm}$ diameter, others coalescent in the periphery with typical subpleural triangular shape. Interstitial septum thickening resembling a reticular pattern can also be seen. 


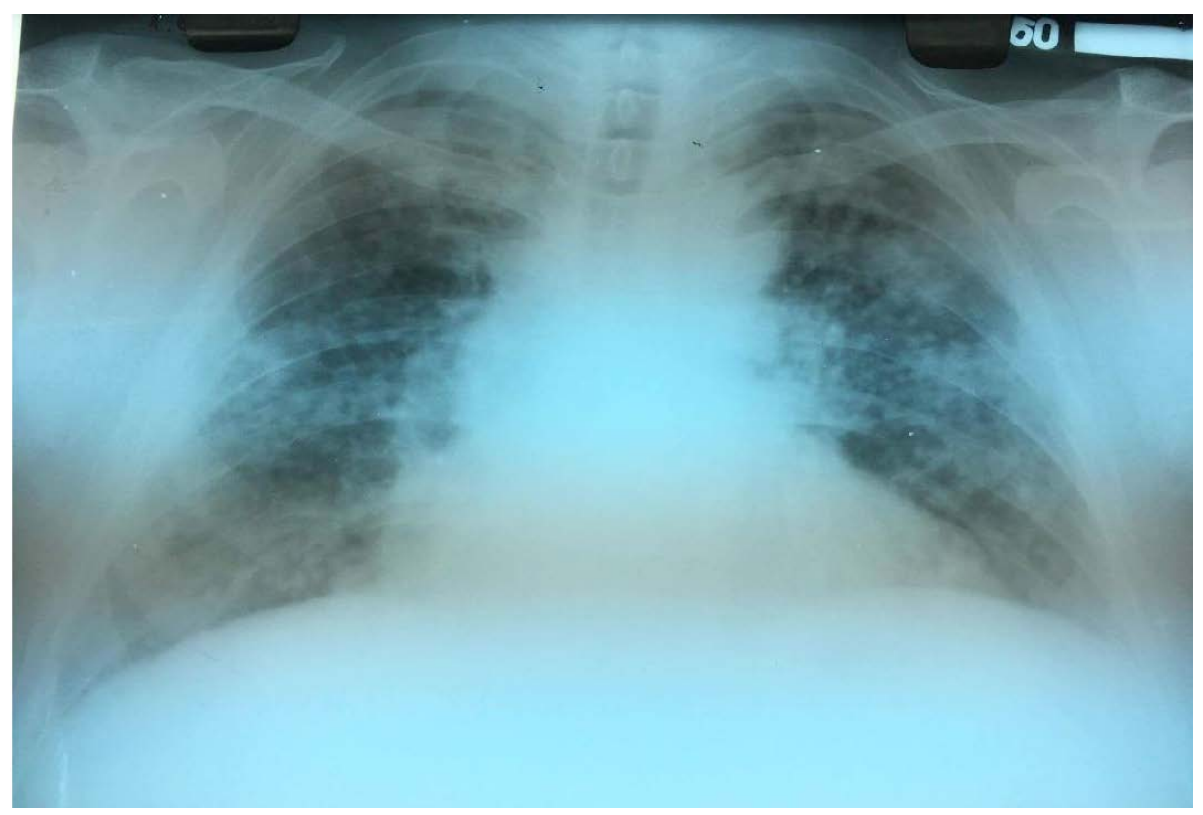

FIGURE 2. Chest X-ray showing silicosis, stage $3 / 3$ r/q B ax, with several round-shape disseminated nodules with a diameter between 3-10 mm and macronodules of 2-3 cm in both pulmonary regions. Also, multiple large mediastinal lymph nodes are present bilaterally.

tion, a chest radiograph was performed, which identified a pulmonary nodular condensation process, for which the patient was guided to the local Pneumology Hospital, to exclude pulmonary tuberculosis. After one month, during which the pulmonary disease had progressed acutely and a positive diagnosis had not been established, an exploratory thoracotomy with pulmonary biopsy was conducted. Macroscopically, the biopsied tissue was of brown-blackish color, and the histopathological examination revealed a granulomatous pulmonary reaction - most likely an anthracotic process without signs of malignancy. However, the results could not completely rule out neither sarcoidosis nor pulmonary tuberculosis. Further investigations included a bronchoalveolar lavage, which described multiple coniophages, siderophages, and macrophages with no fungal elements; the evaluation of ACE serum levels, which was in the normal range; functional ventilatory testing, which revealed moderate restrictive ventilatory dysfunction; and imaging studies including a repeated chest $\mathrm{X}$-ray and thoracic CT (Figure 1A, 1B), which showed images suggestive for silicosis. The diagnosis comprised stage $2 / 3$ $\mathrm{r} / \mathrm{q} \mathrm{B}$ ax silicosis and seropositive RA, and the final diagnosis of Caplan's syndrome was established.

In the following 18 months, the patient presented 4 acute pulmonary infections, with acute respiratory failure, requiring emergency hospitalization, with systemic course of antibiotics and corticosteroids, in addition to his chronic treatment. Each infectious episode was associated with a decreased capacity of healing, requiring longer periods of recovery. The radiological imaging showed an obvious interstitial pulmonary disease progression (Figure 2), thus leading to changing the stage of silicosis diagnosis from $2 / 3$ $\mathrm{r} / \mathrm{q} \mathrm{B}$ ax to $3 / 3 \mathrm{r} / \mathrm{q} \mathrm{B}$ ax.

The second case reports a 50-year-old male patient, nonsmoker, who has been a foundry worker for 17 years, with exposure cessation 15 years ago. From his personal medical history, he had been suspected of tuberculosis in April 2016, when he presented cough with intermittent expectoration, wheezing, shortness of breath, weight loss, and anterior thoracic pain, for which several clinical and paraclinical investigations were performed. The chest $\mathrm{X}$-ray described bilateral bullous emphysema located both apically and in the pulmonary base, medium to high intensity inhomogeneous micronodular and macronodular opacities that are confluent in large mass on both medio-superior pulmonary areas, having a bilateral pseudotumoral aspect. The interlobar pleura and the pericardium appeared to be drawn in several areas, and bilateral hilar calcifications were present. The intercostal spaces appeared to be enlarged, and the cardiac silhouette appeared to be normal (Figure 3).

A fibrobronchoscopy was performed, which showed extrinsic compression of the right superior pulmonary lobe. The sputum test came back negative for Ziehl-Neelsen staining. A native thoracic CT was performed, which revealed suggestive images for accelerated silicosis with massive progressive fibrosis (MPF) (Figure 4A, 4B, and 4C). 


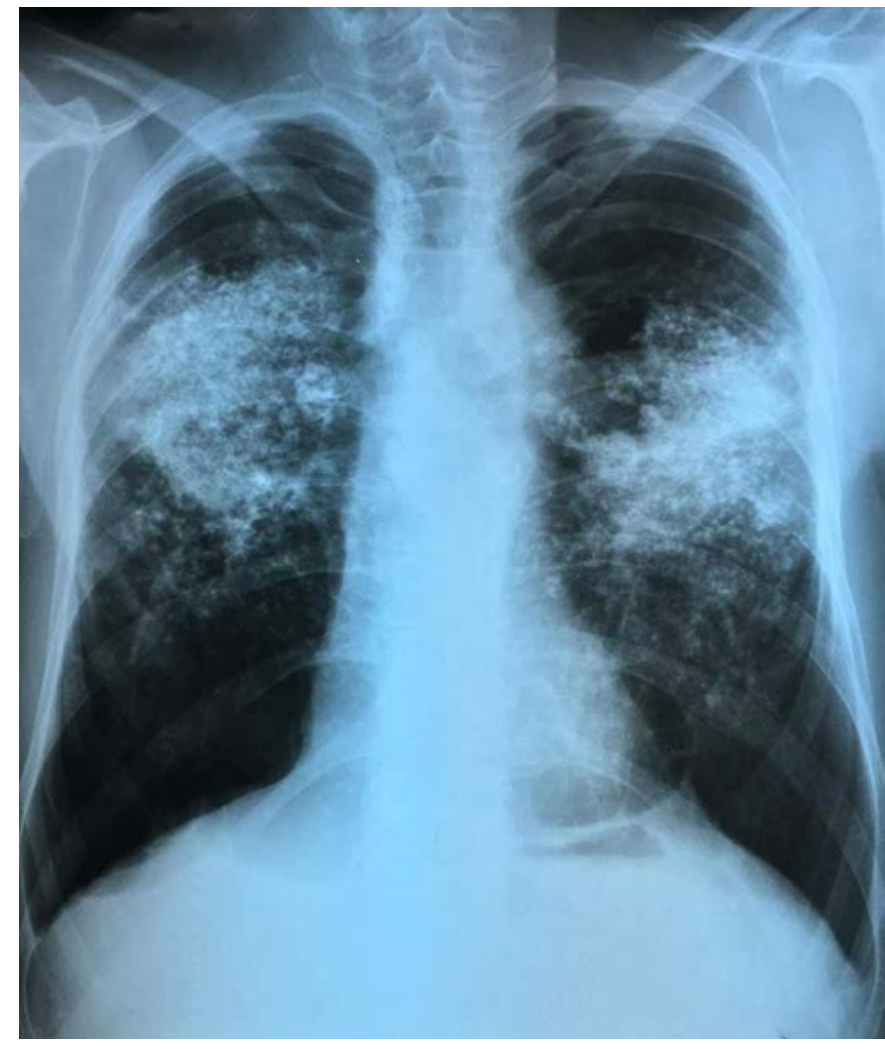

FIGURE 3. Chest X-ray showing Silicosis stage $3 / 3 \mathrm{C}$ q/t $\mathrm{cn}$ em with large bilateral perihilar masses located with irregular dull edges, formed by the confluence of round irregular shaped nodules around $3 \mathrm{~mm}$ in diameter; multiple large mediastinal lymph nodes with eggshell calcification, multiple pleural tractions.

After 2 months, the patient was guided to the Medical Department, presenting for nocturnal paroxysmal dyspnea, effort dyspnea, right intercostal pain, abundant nocturnal transpiration; the symptomatology reappeared after 6 weeks, for which treatment with large spectrum antibiotics was initiated. To elucidate the etiology of chest pain, a cardiac stress test had been performed, which was suggestive for angina pectoris. The abdominal ultrasound described an inhomogeneous granular modified spleen, modified splenic contour, a hypoechogenous formation on the superior portion, which led to the decision to evaluate the serum levels of ACE, which came back slightly elevated, thus revealing the progressive state of the pneumoconiosis. The final diagnosis was confirmed: silicosis stage $3 / 3 \mathrm{C} \mathrm{q} / \mathrm{t} \mathrm{cn}$ em, with massive progressive fibrosis.

After 1 year since his first presentation, the patient had presented in the Emergency Room once again, with the following complaints: fever, cough, dyspnea during rest, which were suggestive for a new respiratory tract infection (third in a row) with acute respiratory failure, for which triple antibiotic therapy was administered, to cover a possible anaerobe infection. After the large spectrum antibiotherapy, corticosteroids in association to the chronic treatment, the patient had partially recovered.

\section{DISCUSSIONS}

In both cases, there were difficulties in establishing a proper diagnosis of silicosis, ${ }^{9}$ for which supplementary investigations were needed, including fibrobronchoscopy, multiple thoracic CT scans, bronchoalveolar lavage, and even lung biopsy. ${ }^{10}$

The first case illustrates an episode of acute progression of the existing lung pathology (an acute evolution of silicosis, probably in the context of the autoimmune condition under treatment with Methotrexate). Bronchoalveolar lavage might contribute to a proper diagnosis in a timely fashion, for initiating therapeutic measures that could lead to cessation of this rapid disease progression..$^{1,2}$
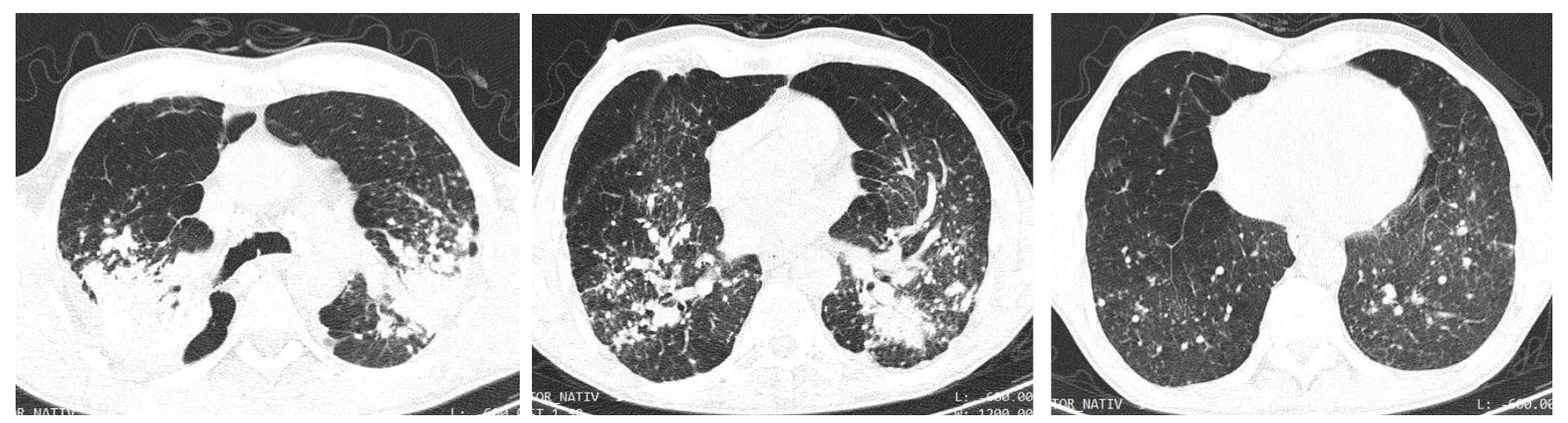

FIGURE 4. Native thoracic CT suggestive for accelerated pneumoconiosis. Bilateral mediobasal and parahilar multiple calcified conglomerate nodular lesions, as large focal masses. Pleural thickening and traction at the surface of the lesions. A reticular pattern, especially at the left basal area, surrounded by emphysematous tissue. The mediastinal window confirming the bilateral presence of calcified lymphadenopathy with macronodular aspect in the perihilar region of the mediastinum. 
In the second presented case, the bronchoalveolar lavage had no therapeutic indications, because the massive pulmonary fibrosis on both lungs was suggestive for an organized form of pneumoconiosis. Like other cases cited in the literature, the second patient presented elevated serum levels of ACE, which indicate the progressive state of the disease. ACE levels can increase in other types of pneumoconiosis, including siderosis, anthracosis, or sarcoidosis. ${ }^{10}$

It is noticeable that both patients presented multiple intercurrent infections in the medium- and long-term follow-up, and both required large-spectrum antibiotics in association to corticosteroids and the chronic treatment. All these infectious episodes can lead to a longer recovery period and decrease the overall clinical and respiratory status of such patients, leading to reserved prognosis after several infectious episodes.

\section{PARTYCULARITY OF THE CASES}

The first case contours a young patient with Caplan's syndrome, a rare association between a rheumatologic disease and an accelerated form of occupational pulmonary disease. In both cases, the respiratory diseases presented late onsets, after more than 15 years of exposure, which is not unusual, but both patients showed a fast progression into massive pulmonary fibrosis, which in association to multiple intercurrent respiratory infections, can lead to worsening of patient recovery and outcomes.

\section{CONCLUSIONS}

Symptoms of occupational pulmonary disease can occur years after cessation of exposure, and diagnosis can be difficult especially in patients presenting with concomitant acute respiratory infections and secondary respiratory failure. The rare association of autoimmune diseases with occupational pulmonary disease can lead to worsening of patient outcomes, and acceleration towards massive progressive pulmonary fibrosis. Several invasive diagnostic procedures such as lung biopsy and bronchoalveolar lavage, in association with non-invasive imaging methods are needed for proper diagnosis and patient management.

\section{CONFLICT OF INTEREST}

Nothing to declare.

\section{REFERENCES}

1. Greenberg Ml, Waksman J, Curtis J. Silicosis: A Review. Dis Mon 2007;53:394-416

2. Leung CC, Yu IT, Chen W. Silicosis. Lancet. 2012;379:2008-2018.

3. Ferreira AS, Moreira VB, Ricardo HM, Coutinho R, Gabetto JM, Marchiori E. Progressive massive fibrosis in silica-exposed workers. High-resolution computed tomography findings. J Bras Pneumol. 2006;32:523-528.

4. L'Abbate N, Di Pierri C, Nuzzaco A, Caputo F, Carino M. Radiological and functional progression in silicosis. Med Lav. 2005;96:212-221.

5. Di Pierri C, Nuzzaco A, Carino $M$, et al. Changes in the radiologic and spirometric profile in subjects with pulmonary silicosis. G Ital Med Lav Ergon. 2003;25:163-164.

6. Bouros E, Filidou E, Arvanitidis K, et al. Lung fibrosis-associated soluble mediators and bronchoalveolar lavage from idiopathic pulmonary fibrosis patients promote the expression of fibrogenic factors in subepithelial lung myofibroblasts. Pulm Pharmacol Ther. 2017;46:78-87.

7. Baur X, Heger M, Bohle RM, et al. Guideline (S2k, AWMF) of the Deutsche Gesellschaft für Pneumologie und Beatmungsmedizin and the Deutsche Gesellschaft für Arbeitsmedizin und Umweltmedizin "Diagnostics and Expert Opinion in the Occupational Disease No. 4101 Silicosis (Including Coal Worker's Pneumoconiosis)". Pneumologie. 2016;70:782-812.

8. Fernández Álvarez R, Martínez González C, Quero Martínez A, Blanco Pérez JJ, Carazo Fernández L, Prieto Fernández A. Guidelines for the diagnosis and monitoring of silicosis. Arch Bronconeumol. 2015;51:86-93.

9. De Capitani EM, Schweller M, Silva CM, Metze K, Cerqueira EM, Bértolo MB. Rheumatoid pneumoconiosis (Caplan's syndrome) with a classical presentation. J Bras Pneumol. 2009;35:942-946.

10. Pandey JK, Agarwal D. Biomarkers: A potential prognostic tool for silicosis. Indian Journal of Occupational and Environmental Medicine. 2012;16:101107. 